\title{
Spontaneous rupture of abdominal aorta - an unusual manifestation of an idiopathic cystic medial necrosis (Gsell-Erdheim syndrome) and literature overview
}

\author{
Aleksander Lukasiewicz', Andrzej Marszalek ${ }^{2,3}$ \\ 'Doctor Wladyslaw Bieganski Regional Specialty Hospital in Grudziadz, Poland \\ ${ }^{2}$ Chair of Pathomorfology, Collegium Medicum in Bydgoszcz, Nicolaus Copernicus University in Torun, Poland \\ ${ }^{3}$ Chair of Oncologic Pathology and Prophylactics, Poznan University of Medical Sciences and Greater Poland Cancer Center, \\ Poznan, Poland
}

\begin{abstract}
In this report, a case of a male, 40-year-old patient who suffered from spontaneous rupture of undilated abdominal aorta, a manifestation of idiopathic cystic media necrosis (Gsell-Erdheim syndrome), is presented. Spontaneous ruptures of medium-to-large arteries observed in this syndrome are a consequence of elastic fibres degeneration and glucose-amino-glycans (GAG) depositions in the arterial media. Gsell-Erdheim syndrome is also observed in a large portion of spontaneous aortic dissections. Histopathological criteria for the diagnosis of this disease, as well as clinical implications for current treatment, especially endovascular procedures in the aortic dissections, are discussed.
\end{abstract}

Key words: cystic medial necrosis of aorta, complications, aortic diseases

Acta Angiol 2016; 22, 1: 16-19

\section{Introduction}

Idiopathic cystic medial necrosis (CMN) is a rare phenomenon where idiopathic elastolysis and GAG deposition in the medial layer of elastic arteries occurs. Microscopic features of this syndrome were described by Gsell and Erdheim in the thirties of the last century [1, 2]. At first elastic fibres swelling and hypochromia occur, then elastin fibres ribbon-like deformation and rupture are found. Finally, diffused elastic lamina destruction with cystic like forms and GAG depositions are observed [3].

The most common manifestation of the syndrome is aortic dissection, however other locations of the disease were also described [4-10]. To our knowledge, non-dilated abdominal aortic rupture such as we have observed in a patient treated at our facility was never previously reported and related to the idiopathic CMN. The aim of this report is to increase the awareness among vascular specialists of this rare but often fatal entity and discuss potential implications in the endovascular treatment of aortic dissections.

\section{Case study}

Previously healthy, male patient, aged 40, presented at a small regional hospital complaining on mild abdominal pain. He negated trauma. Physical examination revealed a moderate tenderness in the mid-abdomen with no other signs. Abdominal ultrasound and X-ray examinations at admission revealed no abnormalities. The patient was admitted to the surgical ward for further evaluation. In the course of the disease sud-

Address for correspondence: Aleksander Lukasiewicz, Doctor Wladyslaw Bieganski Regional Specialty Hospital in Grudziadz, Rydygiera 15/17, 86-300 Grudziadz, Poland, e-mail: alukasiewicz@wp.pl 


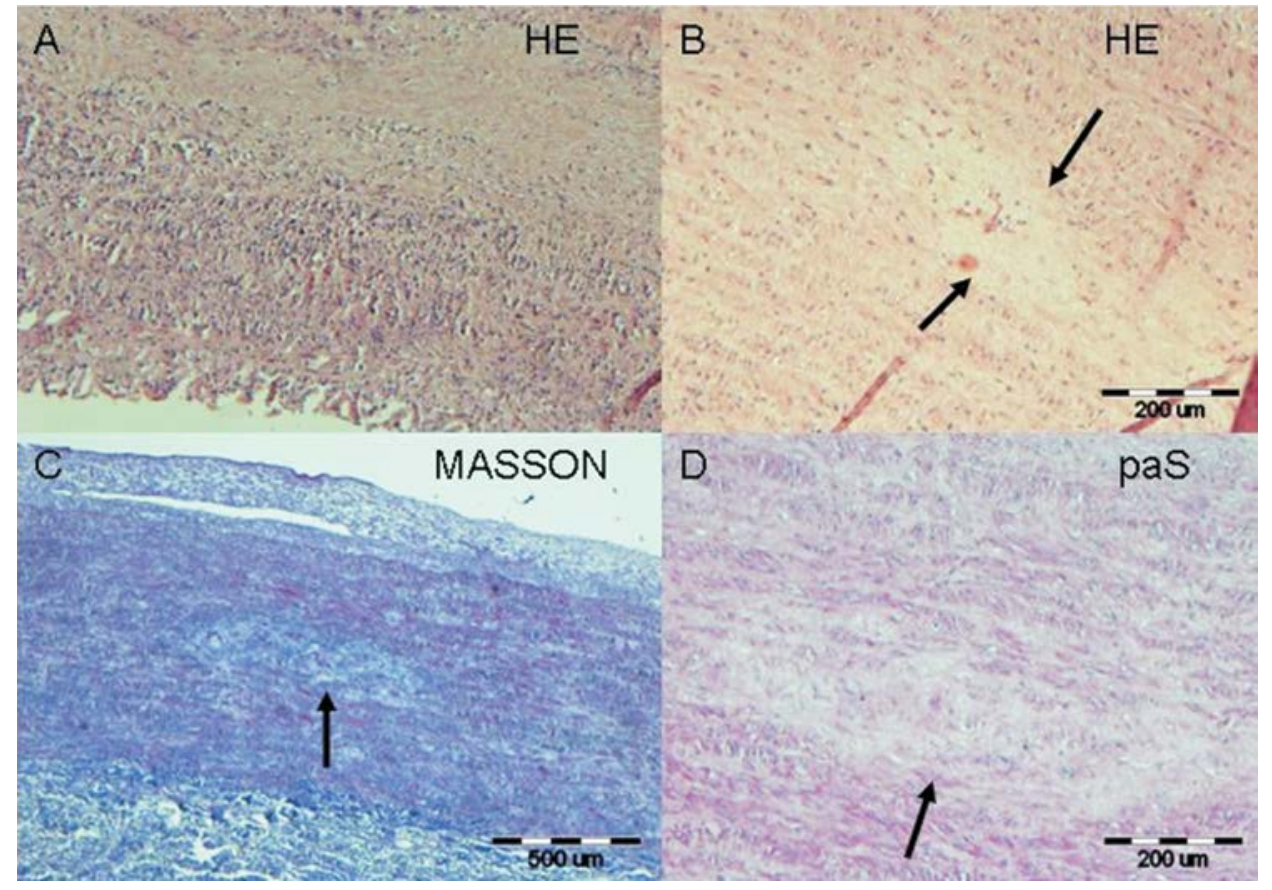

Figure I. Aortic wall specimens: A. There is well visible inflammatory infiltration of aortic wall at the site of rupture. $\mathrm{H} \& \mathrm{E}$, primary objective mag. I0X; B. Focal media destruction with depletion of elastic fibres and lack of cells (between arrows). H \& E, primary objective mag. 10×; C. Focal depletion of smooth muscle cells in the media (thick arrow). Masson trichrome, primary objective mag. I0×; D. Focal amorphic GAG deposition in the media (arrow). paS reaction, primary objective mag. $10 \times$

den hypotension and tachycardia occurred suggesting internal bleeding. Abdomen ultrasound revealed aorta surrounding fluid in the retroperitoneal space. Haemoglobin level decreased (from initial $15 \mathrm{~g} / \mathrm{dL}$ to $9 \mathrm{~g} / \mathrm{dL}$ ). The patient was promptly transferred to the vascular department with suspected abdominal aorta rupture. Emergency laparotomy revealed longitudinal rupture of otherwise macroscopically intact aorta. The lesion involved a $3 \mathrm{~cm}$ portion of aorta and ended approximately $5 \mathrm{~cm}$ above the aortic bifurcation. The ruptured portion of aorta was excised and $16 \mathrm{~mm}$ diameter tube graft (Unigraft, B. Braun, Germany) was sutured to the macroscopically healthy aortic wall. On the $5^{\text {th }}$ post-operative day suddenly a haemorrhagic shock developed. The abdomen was explored suspecting leaking anastomosis. Instead, a new aorta rupture below previous implant was found. A bifurcated $16 / 8 \mathrm{~mm}$ prosthesis (Unigraft, B. Braun, Germany) was sutured proximally to the graft and distally to the common femoral arteries (polypropylene, Surgipro ${ }^{\mathrm{TM}}$, Covidien, Ireland). The distal stump of the aorta was closed using 0 polypropylene suture (Surgipro ${ }^{\mathrm{TM}}$, Covidien, Ireland). Samples of the changed and ruptured aorta were taken for microscopic haematoxylin-eosin evaluation. Further postoperative course was uneventful and on the $10^{\text {th }}$ post-operative day patient was discharged. The pathologist described histological changes in the aorta specimens as an unspecific aortitis with mononuclear infiltration (Fig. IA). Considering this, oral antibiotic (Ciprofloxacin $500 \mathrm{mg}$ twice daily) was prescribed for further 3 months.

Eighteen months later the patient presented with purulent fistula in the inguinal region. Pseudomonas aeruginosa strain was isolated. The whole infected vascular graft was replaced with silver releasing prosthesis (Silverguard ${ }^{\circledast}$, Intervascular, USA) in the anatomic position. Intravenous and subsequent oral antibiotic according to the culture and antibiogram was administered for additional 3 months. The postoperative course was uneventful and the patient was discharged. After further 19 months he presented again, at this time with pseudoaneurysm of the right distal anastomosis. The patient was qualified to the surgical treatment. During the operation, considering previous graft infection, swabs and arterial wall samples were collected for culture. Additionally, marked weakening of the wall of the common femoral artery was observed. Samples of the artery wall were harvested and sent for further histologic evaluation. Surgical reconstruction of the anastomosis was performed using $8 \mathrm{~mm}$ PTFE prosthesis (Gore-tex ${ }^{\circledR}$, W. L. Gore \& Associates, Inc, USA). The postoperative course was uneventful and patient was discharged on the $7^{\text {th }}$ day post-op. Bacterial culture results were negative. Haematoxylin/eosin staining re- 


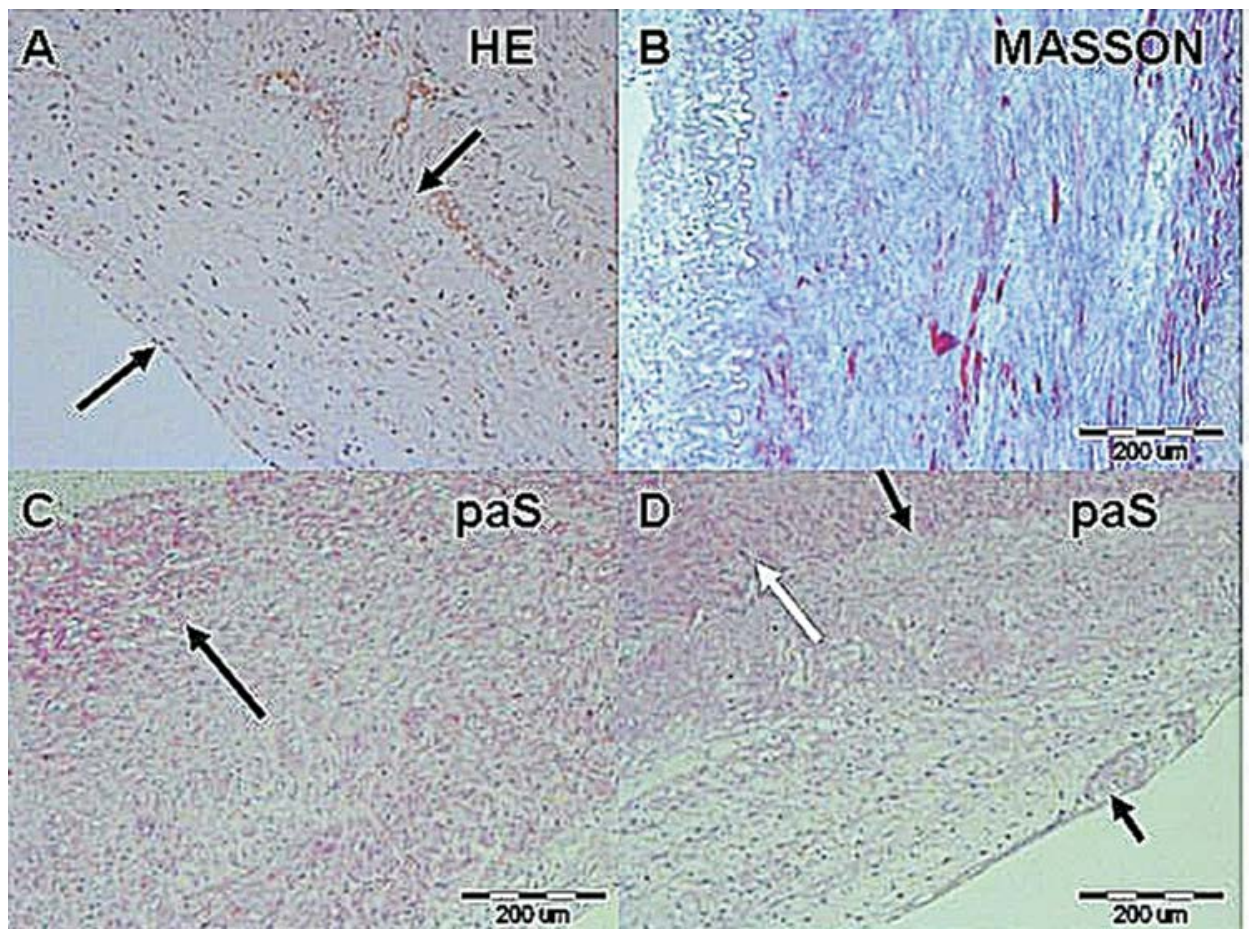

Figure 2. Femoral artery specimens: A. Marked intimal thickening in the femoral artery (between arrows). $\mathrm{H} \& \mathrm{E}$, primary objective mag. 10X; B. Diffused depletion of smooth muscle cells in the media. Masson trichrome, primary objective mag. $10 \times$; C. Extensive GAG's depositions in the media (arrow). paS reaction, primary objective mag. I0×; D. Thickened intima (between black arrows) and GAG depositions (white arrow) in the arterial wall. paS reaction, primary objective mag. 10X

vealed marked elastic lamina disorganization and intimal thickening. Further Mason staining and paS reaction showed elastic fibres diffuse lysis and GAG depositions in the media (Fig. 2A-2D). Considering these findings, the specimens collected at the second operation during initial presentation were re-evaluated. Mason staining and $\mathrm{paS}$ reaction confirmed that foci of media disintegration with loss of muscle cell, disorganization of elastic fibres and GAG depositions were already present in the aortic media during initial presentation (Fig. IB-ID). These changes were less obvious than in the femoral artery but apparently sufficient to support the diagnosis of CMN as the cause of the aortic wall rupture. Inflammatory infiltration was also confirmed but the clinical course indicated that it was the effect rather than the cause of aortic wall rupture.

\section{Discussion}

Cystic medial necrosis (Gsell-Erdheim syndrome) is a rare entity in the clinical setting. The first description came from Gsell and Erdheim (Germany) who linked spontaneous aortic dissections with microscopic features of aortic media degeneration. These observations were soon confirmed in the USA [I I]. As the knowledge of the distinct microscopic changes in CMN grew, it became apparent that it might involve different parts of vascular system. Ascending aortic, Valsalva sinus, coronary, iliac and visceral artery aneurysms were all described as manifestations site of CMN [4-10].

Current knowledge of the pathologic changes in $\mathrm{CMN}$ delineates distinct features found in both intima and media of the artery. In the intima, a hyperplasia due to accumulation of extracellular matrix components occurs, presumably as a secondary response to the changes in the media. This was present in the specimens from our patient.

The most important changes however are found in the media and consist of medianecrosis and anuclear areas, elastolysis and cystic spaces filled with glycoaminoglycans depositions. The degree of elastolysis can be assessed according to the Shlatmann classifications: I grade - less than 5 foci of elastic fragmentation (in one field of view, magnification $200 \times$ ), every focus includes 2-4 adjacent elastic membranes, orientation of smooth muscle cells (SMC) is preserved; II grade -5 or more foci of fragmentation (in one field of view, magnification $200 \times$ ), foci may be dispersed in the media or fused, orientation of SMC is preserved; III grade - fragmentation of more than 5 adjacent elastic membranes, independently of the amount of foci (in one field of view, magnification $200 \times$ ), orientation of SMC is damaged [12]. Serial aortic wall histopathological image analysis revealed that the media changes were observed in the entire aorta [3]. Considering the clinical course of our patient we might 
speculate that the disease may affect simultaneously different regions of arterial tree and eventual rupture occurs in its the most affected part.

Most often CMN is mentioned in the context of possible causes of aortic dissections. Aneurysm descriptions are also frequent. The largest report to our knowledge came from Ukraine and consisted of 24 cases of spontaneous aortic dissections due to CMN [3]. After thorough search of Medline and Pubmed databases it seems that a case of spontaneous normal size abdominal aortic rupture due to $\mathrm{CMN}$ has never been previously reported. It must be stressed that no macroscopic changes other that local rupture were observed in the aorta wall at the initial operation. Aorta was of normal size and the vessel wall thickness and structure encouraged surgeon to perform only limited aortic reconstruction which led to further dramatic events in the course of the disease. Histology of the specimens obtained sequentially from our patient indicates that arterial wall elastolysis might be a progressive pathology gradually involving not only aortic wall but also more peripheral arteries. This observation was confirmed by other authors [13]. In our patient microscopic examination of superficial femoral artery revealed extensive elastic fibres degradation and replacement by GAG's. This implies that the patient is in constant danger of further arterial ruptures and anastomotic complications and therefore requires lifelong follow-up.

It might be discussed if the endovascular stentgraft repair would be more appropriate in our patient. In our opinion, urgent laparotomy was the only reasonable choice we had at the moment of patient's first presentation. It was dictated by two factors: patient's condition and lack of aortic dilatation and no signs of aortic dissection. Only laparotomy would be suitable if there was some other extraaortic source of bleeding.

Current trends in the treatment of aortic type B dissection favour endoluminal techniques with a stentgraft implantation to exclude the entry site. Considering the fact, that $C M N$ is observed in most aortic dissection cases and the progressive nature of the disease, we are convinced that the surveillance of patients with endovascular stentgraft devices should be especially vigilant. The progressive aortic media degeneration may lead to further aortic wall weakening and dilatation causing the type I endoleak and its catastrophic consequences.

\section{Conclusions}

The rupture of normal sized abdominal aorta must be included in the spectrum of potential cystic medial necrosis symptoms. The disease is progressive in nature and affects not only large elastic arteries but also smaller muscular ones. It leads to the diffuse arterial wall weakening and possible complication. Further efforts on elucidating the aetiology of the cystic medial necrosis are required to identify population at risk. Life-long surveillance is mandatory in all patients with diagnosed cystic medial necrosis.

\section{References}

I. Erdheim J (1930) Medianecrosis aortae idiopathica cystica. Virchows Arch; 276: 764-766.

2. Gsell $O$ (1928) Wandnekroses der als selb standige Erkrankung und ihre Bezeihung zur Spontanrupteur. Virchows Arch; 27: I-36.

3. Zerbino D, Kusik J, Havrilyuk E (2005) Medianecrosis of the aorta (MNA)-Gsell-Erdheim syndrome: main histopathological features. Pol J Pathol; 56: 75-79.

4. Attenhofer $\mathrm{CH}$, Vogt $P R$, von Segesser LK, Dirsch OR, Ritter M, Jenni $R$ (1996) Leaking giant aneurysm of the aortic root due to cystic medial necrosis with pericardial tamponade mimicking type-A aortic dissection. Thorac Cardiovasc Surg; 44: 103-104.

5. Yamamoto K, Honma T, Kazurayama M et al (1993) A case of spontaneous rupture of the ascending aorta. Kokyu To Junkan; 4I: 697-700.

6. Takeda K, Matsumiya G, Nishimura M, Matsue H, Tomita Y, Sawa $Y$ (2007) Giant circumflex coronary artery aneurysm associated with cystic medial necrosis in a non-Marfan patient. Ann Thorac Surg; 83: 668-670.

7. Lau H, Cheng SW, Lam KY. (200I) Carotid artery aneurysm secondary to cystic medial necrosis. J R Coll Surg Edinb; 46: 173-175.

8. Bechstein WO, Dralle H, Kemnitz J (1989) Splenic artery aneurysm in cystic media necrosis (Erdheim-Gsell). Spleen-saving surgical therapy. Chirurg; 60: 634-637.

9. Kato T, Takagi H, Kawai N, Sekido Y, Umemoto T (2009) Ruptured isolated external iliac artery true aneurysm associated with cystic medial necrosis: report of a case. Surg Today; 39: 705-709.

10. Moritz AR (1932) Medionecrosis Aortae Idiopathica Cystica. Am J Pathol; 8: 7I7-734.

II. Abdool-Carrim AT, Robbs JV, Kadwa AM, Kenoyer G, Cooper K (1996) Aneurysms due to intimomedial mucoid degeneration. Eur J Vasc Endovasc Surg; I I: 324-329.

12. Schlatmann TJM, Becker AE. Pathogenesis of dissecting aneurysm of aorta. Comparative histopathologic study of significance of medial change. Am J Cardiol; 39: 21-26.

13. Ochiai Y, Kurisu K, Kajiwara T, Kumeda H, Tominaga R (2004) Extended total arch replacement for type $B$ aortic dissection with ascending aneurysm following healed aortic dissection. Jpn J Thorac Cardiovasc Surg; 52: 8I-83.

14. Bastos Gonçalves F, Metz R, Hendriks JM et al (2010) Decision-making in type- $B$ dissection: current evidence and future perspectives. J Cardiovasc Surg (Torino); 51 : 657-667. 